\title{
A ATUAÇÃO DOS ENFERMEIROS EGRESSOS DO CURSO DE ESPECIALIZAÇÃO EM OBSTETRÍCIA NO NORDESTE DO BRASIL - DA PROPOSTA À OPERACIONALIZAÇÃO
}

The role of nurses graduated at the course of expertise in obstretics, in the northeast of Brazil - from the proposal up to the operacionalization.

La actuación de las enfermeras egresas del curso de especialización en el nordeste de Brasil - desde la propuesta hasta la operación

\author{
Arabela Antônia Nery de Melo Costa ${ }^{1}$
}

Janine Schirmer ${ }^{2}$

\section{RESUMO}

Com o objetivo de detalhar a atuação de enfermeiros após especialização em Obstetrícia na Região Nordeste do Brasil e os benefícios dessa atuação consubstanciados em premiações para a instituição em que trabalhavam, procedeu-se a estudo transversal, descritivo, exploratório, entre maio de 2006 e março de 2007, em duas fases: a primeira com entrevista estruturada para 127 $(67,2 \%)$ dos 345 egressos da especialização, residentes em nove estados do Nordeste, e a segunda com contato telefônico via web a $56(98,2 \%)$ enfermeiros. Constatou-se que $114(90,5 \%)$ egressos atuavam na assistência, $50(39,7 \%)$ na administração e 47 (37,3\%) na docência de enfermagem. Só em Pernambuco, os egressos lecionavam a residentes de enfermagem. Predominou a concessão da premiação pelo Ministério da Saúde a instituições com egressos. Concluiu-se que cursos de especialização em enfermagem obstétrica, financiados pelo Ministério, ensejaram ganhos a egressos e instituições, permitindo melhor assistência, resultando em premiações pela implantação de novos modelos assistenciais.

Palavras-chave: Enfermeiras obstétricas. Prática profissional. Área de atuação profissional.

\begin{abstract}
With the aim of clarifying in detail the role of nurses after the specialization in obstetrics, in the nor theast of Brazil, and the benefits of this substantial performance in awards to the institution where they used to work. A cross-sectional, descriptive and exploratory study was proceeded between May 2006 and March 2007, divided in two steps: The first one applying a structured interview for $127(67,2 \%)$ from the 345 graduated at the specialization, residents from the nine states located in the nor theast, and the second one was through telephone contacts via web to $56(98,2 \%)$ nurses. We noticed that $114(90,5 \%)$ from the graduated, worked in assistance, $50(39,7 \%)$ at the administration and $47(37,3 \%)$ teaching in nursing. Only in Pernambuco, the graduated used to teach the residents in nursing. The granting of the award by the Ministry of Health to the institutions with graduates was predominant. It was concluded that specialization courses in obstetrics nursing have contributed to provide gains to the graduates and to the institutions, allowing a better assistance, resulting in awards for the implementation of new models for care assistance.
\end{abstract}

Keywords: Obstetric Nurses. Professional Practice.Area of Professional Activity.

\section{Resumen}

Para detallar la actuación de las enfermeras después de la especialización en Obstetricia, en el Nordeste de Brasil y los beneficios de esta acción representados por premiación a la institución donde trabajaban, se realizó el estudio transversal, descriptivo, exploratorio, entre mayo de 2006 y marzo de 2007 en dos fases: la primera, con entrevista estructurada a 127 $(67,2 \%)$ de los 345 graduados de especialización, que vivían en nueve estados del Nordeste; la segunda, con contacto por teléfono a través de web con 56 (98,2\%) enfermeros. Se constató que $114(90,5 \%)$ graduados trabajaban en la atención, 50 $(39,7 \%)$ en la administración y 47 (37,3\%) en la enseñanza de enfermería. Sólo en Pernambuco, los graduados estaban enseñando en residencias de enfermería. La premiación concedida por el Ministerio de la Salud predominó para instituciones con graduados. Se concluyó que los cursos de especialización en enfermería obstétrica, financiados por el Ministerio, oportunizaron los egresos e instituciones, permitiendo mejor asistencia, resultando en premiación por la implementación de nuevos modelos de atención.

Palabras clave: Enfermeras Parteras. PrácticaProfesional. Áreas de actividad profesional

${ }^{1}$ Enfermeira Obstetra. Professora Adjunta da Universidade de Pernambuco - UPE. Doutora em Ciências. Coordenadora do Curso de Especialização em Enfermagem Obstétrica da UPE. Recife-PE. Brasil. E-mail: arabela_costa@uol.com.br; ²Enfermeira Obstetra. Professora Titular da Escola Paulista de Enfermagem. Coordenadora do Programa de Pós-Graduação em Enfermagem da UNIFESP. São Paulo - SP. E-mail: schirmer.janine@unifesp.br 


\section{INTRODUÇÃO}

A partir de 1985, a Organização Mundial de Saúde iniciou um debate mundial sobre a mortalidade materno-fetal, que atingia cifras alarmantes. Em 2003, as estimativas mundiais eram de 120 milhões de gravidezes, das quais mais de um milhão de mulheres morriam em consequência de complicações; mais de 50 milhões eram acometidas por enfermidades ou incapacidades graves relacionadas com a gravidez e pelo menos 1,2 milhão de recém-nascidos morriam por complicaç̃̃es durante o parto 1 . Os esforços dos países, dentre os quais o Brasil, passaram a ser redobrados na tentativa de redução dessas taxas.

Em 1985, a Carta de Fortaleza, documento de consenso internacional, baseada no princípio do direito fundamental de a mulher receber cuidados adequados de pré-natal, contemplando os fatores sociais, emocionais e psicológicos², desencadeou um processo de conscientização e aglutinou representantes de grupos e entidades que comungavam preocupações e indignações com a situação do nascer em nossa sociedade, tal como a Rede pela Humanização do Parto e Nascimento, criada em 1993. Havia um propósito de aliar conhecimento técnico e científico, sistematizado e comprovado, a práticas humanizadas de assistência ao parto e nascimento ${ }^{3}$.

A partir do reconhecimento de que a formação de mão de obra qualificada para a assistência ao parto e nascimento e a implantação das recomendações da OMS para prática do parto normal poderiam permitir a redução da mortalidade materno-fetal, foi implementada pelo Ministério da Saúde a proposta de construção de um "Novo Modelo de Atenção à Saúde da Mulher no País".

Ao implantar e financiar Cursos de Especialização em Enfermagem Obstétrica (CEEO), em todo o país, por intermédio da Área Técnica de Saúde da Mulher, a partir de 1999, o Ministério da Saúde reconheceu tacitamente a importância da atuação do enfermeiro obstetra na implementação da Política de Saúde da Mulher ${ }^{4}$, a qual deu origem ao Programa de Humanização no Pré-Natal e Nascimento (PHPN), respaldado pelas Portarias GM/569, 570, 571 e 572/2000 5,6,7

Buscando assegurar a melhoria do acesso da cobertura e da qualidade do acompanhamento pré-natal, da assistência ao parto e puerpério, entre 1999 e 2004, foram ministrados 76 CEE0, em todo o país, especializando 1.366 enfermeiras obstetras ${ }^{8}$.Vinte e dois destes cursos foram ministrados na Região Nordeste, especializando 345 enfermeiras obstetras, distribuídas nas regiões urbanas e rurais. Essa distribuição buscava aumentar a cobertura da assistência com qualidade. Diferente da zona urbana, na qual a maioria dos partos era realizada em ambiente hospitalar, nas zonas rurais, ribeirinhas e lugares de difícil acesso, muitas vezes a única opção era o parto domiciliar, assistido por parteiras tradicionais. Além disso, nessas localidades, os serviços de saúde estavam mal equipados 9 .
A hipótese, que norteou o Ministério da Saúde na concepção desses cursos, foi que enfermeiros obstetras melhor capacitados, exercendo suas atividades com base nas diretrizes do PHPN, poderiam oferecer assistência humanizada ao prénatal, parto e nascimento.

0 exercício do magistério superior por mais de duas décadas, aliado à pesquisa sobre formação e atuação do Enfermeiro Obstetra (EO) no Brasil, nos permitiu supor uma aparente incoerência entre a formação e o exercício profissional, pois parte dos egressos não atuava na assistência, motivando este estudo. 0 objetivo foi detalhar a atuação dos enfermeiros após a especialização em Obstetrícia, com base nas diretrizes do PHPN, na Região Nordeste do Brasil, e os benefícios dessa atuação consubstanciados em premiações para a instituição em que trabalhavam.

\section{MÉTODO}

0 estudo, de caráter descritivo e de corte transversal, foi desenvolvido na Região Nordeste do Brasil e incluiu os estados de Maranhão, Piauí, Ceará, Rio Grande do Norte, Paraíba, Pernambuco, Alagoas, Sergipe e Bahia, ocupando uma área de $1.558 .196 \mathrm{~km}^{2}$, equivalente a $18 \%$ do território nacional, com população de 51.065 .275 habitantes, à época do estudo ${ }^{10}$, quando havia a proporção de 6,2 enfermeiros no exercício profissional por 10 mil habitantes, em $2005^{10}$.

0 estudo foi realizado em duas fases. Na primeira, admitiram-se como critérios de inclusão estar cadastrado como aluno do CEEO do Ministério da Saúde, no período de 1999 a 2004; responder ao contato telefônico da pesquisadora para convite de participação na pesquisa; e responder às questões contidas no questionário semiestruturado. Na segunda fase, foram incluídas as enfermeiras obstetras que, na primeira fase, referiram atuar na assistência ao parto e nascimento e concordaram em participar de entrevista estruturada por meio de telefone via web (voip).

A coleta de dados foi realizada no período de maio de 2006 a março de 2007 e composta por três etapas. Na primeira, foi mantido contato com as coordenadoras dos 22 CEEO, ministrados por 14 Instituições de Ensino Superior da Região Nordeste, para que se obtivesse a relação nominal dos 345 egressos. Na segunda etapa da coleta, foi mantido contato telefônico com todos os egressos, sendo 189 (54,8\%) localizados e convidados a participar, os quais constituíram a população de estudo.

A terceira etapa da coleta esteve constituída por duas fases. Na primeira fase, foram coletadas as respostas de 127 $(67,2 \%)$ egressos, utilizando-se um questionário semiestruturado, com perguntas fechadas e abertas, sobre dados sociodemográficos, formação acadêmica e atuação profissional. Esse total compôs a amostra de conveniência, dado que os participantes da pesquisa se caracterizavam como voluntários. Dos $57(44,9 \%)$ egressos que referiram atuar na assistência ao parto e nascimento, 56 (98,2\%) aceitaram 
participar da segunda fase do estudo, por meio de telefone via web (voip), realizada logo após a aplicação do questionário semiestruturado. Essa fase consistiu na coleta de informações detalhadas sobre atuação profissional, elaboradas a partir das diretrizes propostas pela Área Técnica de Saúde da Mulher do Ministério da Saúde e pela Portaria $n^{\circ} .569$ de $1^{\circ}$ de junho de $2000^{4}$, que criou o Programa de Humanização no Pré-Natal e Nascimento (Quadro 1).

Para detalhamento da atuação profissional, foram consideradas as variáveis: permanência da atuação na assistência ao parto e nascimento, discriminação das modificações realizadas nas práticas da assistência ao parto e nascimento, após a especialização; realização do parto normal; registro do parto realizado, de acordo com a Portaria $n^{0}$. 2815/ 98/GM; preenchimento do Laudo de Enfermagem de Autorização de Internação Hospitalar, de acordo com Portaria $n^{\circ} .163 / 99$ SAS/MS; intervenções desenvolvidas na assistência humanizada ao parto e nascimento de baixo risco, segundo recomendações da OMS/MS, bem como relato de facilidades e dificuldades encontradas para implantar o PHPN nas Instituições onde trabalhavam.

Para caracterização amostral, foram consideradas naturalidade, local de residência, idade, sexo, estado civil, tamanho familiar, grau de parentesco com coabitantes, dados de formação acadêmica e profissional em enfermagem, locais de atuação profissional e tipo de atividade desenvolvida.
Os dados coletados foram compilados em banco de dados e apresentados em forma de tabelas, quadros, gráficos e figuras. Para as variáveis qualitativas, foram apresentadas as distribuições de frequências absolutas (N) e relativas (\%). Para as variáveis quantitativas, foram utilizadas como medidasresumo: média, mediana, além de erro-padrão e valores mínimo e máximo, para indicar a dispersão. Nas comparações, para verificar semelhança de distribuição das diversas variáveis entre os diferentes grupos avaliados, empregou-se o teste QuiQuadrado ou o teste exato de Fisher (F), quando necessário, considerando, em ambos, nível de significância de 5\%.

$\mathrm{Na}$ análise de contingência, em que houve evidência de associação estatística de mais de duas categorias de respostas, foi utilizada a análise de resíduo para identificação dos pares de categorias que correspondiam a um valor maior que 0 esperado, ou seja, quais categorias se diferenciavam entre os grupos. Um valor do resíduo padronizado $\left(\mathrm{z}_{\text {res }}\right)$ maior que 1.96 indicou significância estatística na casa tabular, em nível de significância de $5 \%$. Nos gráficos, foram representados os resultados das características para as quais foi identificada significância estatística $(p<0,05)$.

Este estudo foi registrado e aprovado pelo Comitê de Ética em Pesquisa Envolvendo Seres Humanos da UNIFESP/ EPM, sob o protocolo CEP 1573/04, atendendo às exigências da Resolução 196/96.

Quadro 1-Distribuição dos quantitativos de egressos de especialização em obstetríiasegundo local deformaçãoe participação dasfases do estudo-Brasil, 2006-2007

\begin{tabular}{|c|c|c|c|c|c|c|c|c|c|}
\hline 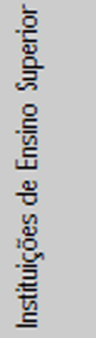 & 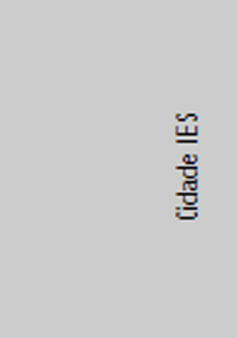 & 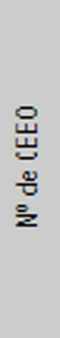 & 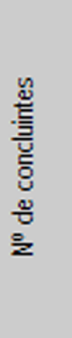 & 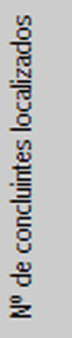 & 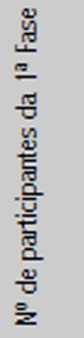 & 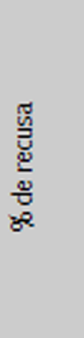 & 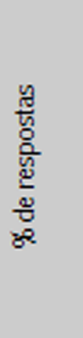 & 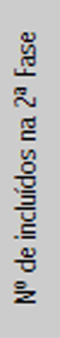 & 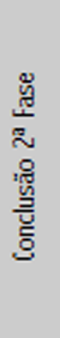 \\
\hline UPE1 & Recife & 02 & 33 & 28 & 20 & 28,6 & 71,4 & 13 & 13 \\
\hline UFBA2 & Salvador & 03 & 40 & 26 & 14 & 462 & 53,8 & 07 & 06 \\
\hline UEFS & Feira de Santana & 02 & 27 & 09 & 06 & 33,3 & 66,7 & 02 & 02 \\
\hline UESC ${ }^{2}$ & |lhéus & 01 & 17 & 07 & 05 & 28,6 & 71,4 & 01 & 01 \\
\hline UESB 5 & Vitória da Conquista & 01 & 20 & 08 & 08 & - & 100,0 & 03 & 03 \\
\hline UFRN 6 & Natal & 01 & 17 & 12 & 05 & 58,3 & 41,7 & 02 & 02 \\
\hline UERN7 & Mossoró & 01 & 18 & 11 & 06 & 45,4 & 54,6 & 02 & 02 \\
\hline UFPB & João Pessoa & 01 & 19 & 07 & 04 & 42,8 & 57,2 & 00 & 00 \\
\hline UEPB? & Campina Grande & 01 & 20 & 14 & 06 & 57,1 & 42,9 & 02 & 02 \\
\hline UFAL'10 & Maceió & 03 & 43 & 20 & 12 & 40,0 & 60,0 & 07 & 07 \\
\hline UFMA 11 & São Luis & 01 & 13 & 08 & 07 & 12,5 & 87,5 & 04 & 04 \\
\hline UFSE12 & Aracajú & 01 & 13 & 11 & 09 & 18,2 & 81,8 & 05 & 05 \\
\hline UECE ${ }^{13}$ & Fortaleza & 01 & 17 & 10 & 08 & 20,0 & 80,0 & 04 & 04 \\
\hline UFP|14 & Teresina & 03 & 48 & 18 & 17 & 5,6 & 94,4 & 05 & 05 \\
\hline TOTAL & 14 & 22 & 345 & 189 & 127 & 32,8 & 67,2 & 57 & 56 \\
\hline
\end{tabular}

Legenda: ${ }^{1}$ Universidade de Pernambuco (estadual), ${ }^{2}$ - Universidade Federal da Bahia, ${ }^{3}$ Universidade Estadual de Feira de Santana (BA), ${ }^{4}$ Universidade Estadual de Santa Cruz (BA), ${ }^{5}$ Universidade Estadual do Sudoeste da Bahia, ${ }^{6}$ Universidade Federal do Rio Grande do Norte, ${ }^{7}$ Universidade Estadual do Rio Grande do Norte, ${ }^{8}$ Universidade Federal da Paraiba, ${ }^{9}$ Universidade Estadual da Paraiba, ${ }^{10}$ Universidade Federal de Alagoas, ${ }^{11}$ Universidade Federal do Maranhão, ${ }^{12}$ Universidade Federal deSergipe, ${ }^{13}$ Universidade Estadual do Ceará, ${ }^{14}$ UniversidadeFederal do Piaú 


\section{RESULTADOS E DISCUSSÃO}

\section{Características sociodemográficas e de formação profissional dos enfermeiros obstetras egressos dos CEEO}

Dos 127 egressos dos CEEO financiados pelo Ministério da Saúde, na Região Nordeste do Brasil, no período de 1999 a 2004, que responderam ao questionário, 123 (96,9\%) referiram ter nascido em estados da Região Nordeste; 3 (2,4\%), na Região Sudeste e 1 (0,7\%) não informou sua origem. Em relação ao estado onde moravam à época da coleta de dados, $126(99,2 \%)$ egressos referiram estados da Região Nordeste. Uma $(0,8 \%)$ enfermeira obstetra residia na cidade de Montes Claros, estado de Minas Gerais, Região Sudeste. Por pertencer ao Bioma 1 Caatinga, essa cidade está sujeita às políticas públicas da Região Nordeste, do que derivou a inclusão dessa egressa na tabulação referente ao estado da Bahia.

As distribuições de naturalidade e local de habitação eram esperadas pelo fato de os cursos terem sido realizados nessa região. Constatou-se que os egressos residiam em 35 municípios dessa região, dos quais $11(31,4 \%)$ localizam-se no estado da Bahia, 5 (14,2\%), em Pernambuco ou no Ceará, e $4(11,4 \%)$, em Alagoas.

A idade dos egressos variou entre 26 e 70 anos, com média de 38,7 $\pm 8,5$ anos. A grande variabilidade etária pode ter derivado do fato de os critérios de seleção para o CEEO não contemplarem idade cronológica, mas, sim, experiência profissional. A maioria dos egressos era do sexo feminino (123; $96,9 \%)$. Na especialidade de obstetrícia, é frequente a presença de enfermeiras, fenômeno que pode estar relacionado com a própria história da arte de partejar, que acontecia no espaço doméstico das famílias, sob domínio das parteiras, até meados dos anos $1950^{11}$. Adicionalmente, a capacitação de recursos humanos para assistência ao parto, fora da profissão médica, contempla quase exclusivamente o sexo feminino, distribuído em várias categorias, com formação e inserção diversificada nos serviços e com atuação técnica heterogênea ${ }^{12}$.

Em relação às características da formação profissional dos egressos dos CEEO (primeira fase do estudo), pôde-se constatar que, de 127 enfermeiros, 115 (90,6\%) referiram ter realizado a graduação em universidades públicas e $12(9,4 \%)$, em instituições privadas. Esse resultado difere do informe do Instituto Nacional de Pesquisas Educacionais Anísio Teixeira $\left(\right.$ INEP) ${ }^{13}$, ao afirmar que, em relação à dependência administrativa, a participação das mulheres é maior nas instituições de ensino superior privadas. Em 2000, as mulheres representavam $41,8 \%$ dos egressos nas instituições privadas contra $39,7 \%$ nas instituições públicas, percentuais que passaram para 45,2\% e 42,6\%, respectivamente, em 2005.

Quanto ao CEE0, todos os egressos foram titulados por instituiçõ̃es de ensino superior, públicas federais ou estaduais, com financiamento do Ministério da Saúde. Os egressos haviam concluído a graduação em Enfermagem entre 4 e 48 anos, com média de 13,8 $\pm 8,0$ anos, o que permite afirmar que a maioria das Instituições atendeu às Diretrizes Curriculares Nacionais para elaboração dos projetos pedagógicos dos $\mathrm{CEE} 0$, e, no processo de seleção dos enfermeiros para esse curso, foi obedecidaa exigência de tempo médio de graduação $0^{4}$.

\section{Atuação profissional das enfermeiras obstetras egressas dos CEEO}

Na Tabela 1, estão apresentados os resultados referentes à atuação profissional dos egressos dos CEEO. Quanto à natureza da instituição em que os egressos declararam trabalhar, 114 (89,8\%) referiram instituições públicas. Este resultado refletiu o recomendado nas Diretrizes Curriculares para elaboração dos CEEO, na qual estava expressa, como proposta do curso, capacitar enfermeiros do SUS33. Um estudo sobre a formação do enfermeiro obstetra no Brasil demonstrou que o vínculo empregatício dos egressos do CEEO, ofertado gratuitamente e apoiado pelo Ministério da Saúde, era do SUS, em $74,0 \%$, exclusivamente e $21,0 \%$ trabalhando em instituições conveniadas ${ }^{11}$. Também uma pesquisa sobre os enfermeiros obstetras egressos da Escola de Enfermagem da Universidade São Paulo demonstrou que a maioria deles também trabalhava em instituições públicas $(67,3 \%)$ e pequeno percentual $(28,3 \%)$ atuava em instituição privada ${ }^{14}$.

Em relação ao número de vínculos empregatícios, identificouse que a maioria dos egressos $(66 ; 52 \%)$ tinha dois vínculos, 39 $(30,7 \%)$ referiram um e 19 (15\%) informaram trabalhar em três instituições. Como a legislação permite até dois vínculos públicos, percebe-se que a prerrogativa legal era majoritariamente utilizada por esse grupo, tal como foi identificado em estudo com egressos de CEEO - modalidade residência - dos quais 92,3\% tinham mais de um vínculo empregatício ${ }^{15}$.

Quanto à área de atuação, dos 127 egressos, 107 (84,3\%) referiram atuar na área da mulher e 18 (14,2) não atuavam, justificando esse fato por: exerćício de cargo administrativo (4;22,2\%) ou falta de oportunidade $(4 ; 22,2 \%)$, dentre outros argumentos. Esse resultado atendeu ao objetivo do CEEO de capacitar enfermeiros para atuação na assistência ao parto e nascimento.

Note-se que há referência de $90 \%$ dos enfermeiros matriculados nos CEEO do Brasil atuarem na área da saúde da mulher, predominantemente (310;51\%) em serviços de atenção ao parto ${ }^{11}$, bem como de 19 (69,2\%) dos 26 egressos dos Cursos de Especialização em Enfermagem Obstétrica e Neonatal - modalidade residência estarem inseridos diretamente na área de formação $0^{15}$.

A dificuldade de obstetrizes e enfermeiras obstétricas se manterem na área da especialidade (obstetrícia) pode ter como causa uma identidade profissional hibrida, que favorece o acúmulo ou desvio de função no exercício profissional, com o deslocamento do enfermeiro obstetra da assistência no pré-natal e no parto para outras áreas da enfermagem, gerando sobrecarga de trabalho, frustração, desmotivação, conformismo e submissão ${ }^{16}$.

No entanto pode-se aventar a hipótese de que essa limitação não seja específica da área de enfermagem obstétrica, ao identificar que entre os egressos dos curso sem cuidados intensivos, apenas $36,8 \%$ dos enfermeiros permaneciam em UTI, 
enquanto a maioria $(60,5 \%)$ não mais atuava nessa área. ${ }^{17}$.

Entre os motivos pelos quais enfermeiros obstetras não atuam na especialidade, têm sido citados: melhor oportunidade de trabalho em outraárea, motivos pessoais diversos e decepção com a atuação em obstetrícia ${ }^{14}$, aspecto este também comprovado em pesquisa no Rio Grande do Sul ${ }^{18}$. Os resultados desta pesquisa, associados aos dados da literatura, sugerem que o abandono da área de atuação é maior por enfermeiros obstetras com maior tempo de formação.

Em relação ao tipo de atividade profissional, 114 (90,5\%) egressos referiram atuar na assistência, 50 (39,7\%), na administração, e 47 (37,3\%), na docência (Tabela 1). Nossos resultados corroboraram a forte associação do trabalho dos enfermeiros com funções de gerenciamento de Unidades ou Instituições, constatada em pesquisa na qual, entre 26 egressos, somente 11 (42,3\%) não exerciam essa atividade. Destes, quatro enfermeiros obstetras atuavam diretamente na assistência em
Centro de Parto Normal, responsabilizando-se pela condução do trabalho de parto e parto, embora tivessem referido função de gerenciamento exercida ao desenvolver supervisão de auxiliares de enfermagem que atuavam no serviço. 0 gerenciamento de serviços de saúde foi atividade principal de $53,6 \%$ dos egressos, sendo $34,6 \%$ em maternidades, revelando que ainda é frequente a cultura de deslocamento de pessoal qualificado em obstetrícia para funções administrativas ${ }^{15}$.

Em outro estudo, $61(66,3 \%)$ egressos informaram ser assistenciais, $34(37,0 \%)$ dos quais trabalhavam em enfermagem obstétrica e 27 (29,3\%), em outras áreas. Dezenove $(20,6 \%)$ egressos eram docentes de enfermagem, sendo que $12(13,2 \%)$ lecionavam enfermagem obstétrica e 7 (7,6\%), conteúdos programáticos de outras áreas do conhecimento. Além desses, 10 egressos mudaram de profissão e $2(2,2 \%)$ declararam-se do $l a r^{14}$.

Tabela 1 - Atuação profissional das enfermeiras obstetras egressas dos CEEO, financiados pelo Ministério da Saúde na Região Nordeste - Brasil, 2006-2007

\begin{tabular}{|c|c|c|}
\hline [aaterísicas hatuacāo prissiond & H & $\%$ \\
\hline \multicolumn{3}{|l|}{ Haurezadingtitucāo } \\
\hline Pública 5155 & 114 & $89,8 \%$ \\
\hline Privada, mas corveriada an SUS & 8 & $6,3 \%$ \\
\hline Privada & 25 & $19,7 \%$ \\
\hline Outro & 8 & $6,3 \%$ \\
\hline \multicolumn{3}{|l|}{ Trabahana área damher? } \\
\hline Sm & 107 & 84,3 \\
\hline 燤 & 18 & 142 \\
\hline Sem Irformą̃ã & 2 & 1,5 \\
\hline \multicolumn{3}{|l|}{ Por quenāotrdadhana áeada mulher?* } \\
\hline Ettá em cago admiristrativo & 5 & 27,8 \\
\hline Trabalhacomo vereadora & 1 & 5,6 \\
\hline Fatt a de oportunidade & 4 & 222 \\
\hline Trabalhaem outra área & 4 & 222 \\
\hline Outras respostas & 4 & 222 \\
\hline \multicolumn{3}{|l|}{ Aividade profssiondt } \\
\hline Docente & 47 & $37,3 \%$ \\
\hline Administraiva & 50 & $39,7 \%$ \\
\hline Asitenda & 114 & $90,5 \%$ \\
\hline Outra & 4 & $3,2 \%$ \\
\hline
\end{tabular}

Considerando as respostas de $46(97,9 \%)$ dos 47 $(37,3 \%)$ egressos que referiram exercer docência, constatouse sua atuação em cursos de auxiliar, técnico, graduação, residência ou pós-graduação em enfermagem nos nove estados da Região Nordeste, em percentuais variando entre $22,2 \%$ e $57,1 \%$ (Tabela 2).

Tabela 2 - Distribuição dos cursos de enfermagem nos quais egressos dos CEEO desenvolviam atividade docente no estado onde residiam - Região Nordeste, Brasil,

\begin{tabular}{|c|c|c|c|c|c|c|}
\hline \multirow[t]{3}{*}{ Etado onde moaram } & \multicolumn{6}{|c|}{ 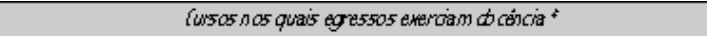 } \\
\hline & 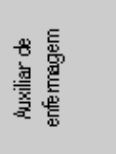 & 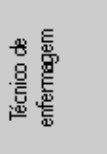 & 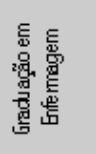 & 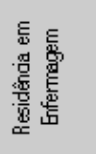 & 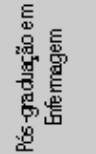 & 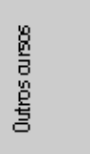 \\
\hline & $n(\mathscr{B})$ & $n(\mathscr{B})$ & $n(8)$ & $n(\mathscr{B})$ & $n(\%)$ & $n(\%)$ \\
\hline $\mathrm{AL}(5 / 12 ; 41,6 \%)$ & $5(100,0)$ & $2(40,0)$ & $1(20,0)$ & - & - & - \\
\hline$B A(18 / 3 ; 545 \%)$ & $6(33,3)$ & $11(61,1)$ & $8(44,4)$ & - & $1(5,6)$ & $2(11,1)$ \\
\hline $\mathrm{CE}(2 / 8 ; 25,0 \%)$ & $1(50,0)$ & $2(100,0)$ & $1(50,0)$ & - & $1(50,0)$ & - \\
\hline$M A(4 / 7 ; 57,19)$ & $3(75,0)$ & $2(50,0)$ & $2(50,0)$ & - & - & - \\
\hline $\mathrm{PB}(412 ; 33,3 \%)$ & $2(50,0)$ & $1(25,0)$ & $2(50,0)$ & - & - & - \\
\hline $\operatorname{PE}(7 / 19 ; 36,808)$ & $2(28,6)$ & $6(85,7)$ & $3(42,9)$ & $4(57,1)$ & - & - \\
\hline$P \mid(5 / 16 ; 31,2 \%)$ & $1(20,0)$ & $4(80,0)$ & $3(60,0)$ & - & - & - \\
\hline $\operatorname{RN}(3 / 11 ; 27,3 \%)$ & $3(100,0)$ & - & $1(33,3)$ & - & - & $1(33,3)$ \\
\hline $\operatorname{SE}(2 / 9 ; 22,2 \%)$ & $1(50,0)$ & $1(50,0)$ & $1(50,0)$ & - & - & - \\
\hline$\rho+a d r$ & 0,301 & 0,250 & 0,882 & 0,042 & 0,497 & 0,970 \\
\hline
\end{tabular}


Pôde-se observar que houve diferença estatisticamente significante entre os diferentes estados exclusivamente na docência de residência em enfermagem, já que somente enfermeiros do estado de Pernambuco $(57,1 \%)$ desenvolviam essa atividade.

Na Tabela 3, estão detalhados os conteúdos ministrados pelos egressos, nos diversos estados nos quais exerciam docência. Observou-se que todos os conteúdos referentes à área da saúde da mulher eram contemplados, sem diferença estatisticamente significante entre os estados, exceção feita aos egressos dos estados de Alagoas e do Rio Grande do Norte, que não lecionavam assistência ginecológica. Este resultado refletiu uma característica da região, onde são poucos os docentes titulados e, por isso, estes geralmente têm a responsabilidade de ministrar vários conteúdos. É importante ressaltar que os egressos docentes reconhecem a importância da formação recebida como fator de segurança, não só na prática assistencial, mas também para o exercício da docência ${ }^{15}$.

Tabela 3 - Distribuição dos conteúdos ministrados pelas egressas nos cursos de enfermagem, segundo estado onde residiam - Região Nordeste - Brasil - 2006-2007

\begin{tabular}{|c|c|c|c|c|c|c|}
\hline \multirow[t]{3}{*}{ Estado on de moram } & \multicolumn{6}{|c|}{ Cursos nos quas egessos exercian docénciat } \\
\hline & 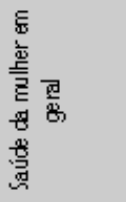 & 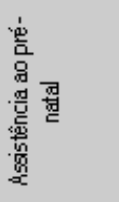 & 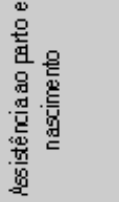 & 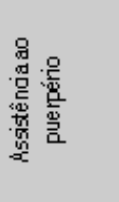 & 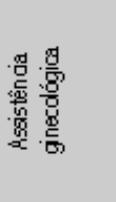 & 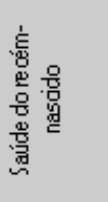 \\
\hline & $n(\%)$ & $n(\infty)$ & $n(\%)$ & $\mathrm{n}(\%)$ & $n(\infty)$ & $n(\$)$ \\
\hline$\overline{\mathrm{AL}}(3 / 12 ; 25,0 \%)$ & $2(66,7)$ & $2(66,7)$ & $2(66,7)$ & $2(66,7)$ & - & $2(66,7)$ \\
\hline$B A(1433 ; 42,4 \%)$ & $14(100,0)$ & $10(71,4)$ & $9(64,3)$ & $8(57,1)$ & $5(35,7)$ & $6(42,9)$ \\
\hline $\mathrm{CE}(3 / 8 ; 37,5 \%)$ & $3(100,0)$ & $3(100,0)$ & $3(100,0)$ & $3(100,0)$ & $3(100,0)$ & $1(33,3)$ \\
\hline$M n(3 / 42,9 \%)$ & $3(100,0)$ & $2(66,7)$ & $2(66,7)$ & $2(66,7)$ & $2(66,7)$ & $2(66,7)$ \\
\hline $\mathrm{PB}(412 ; 33,3 \%)$ & $3(75,0)$ & $1(25,50)$ & $1(25,50)$ & $2(50,0)$ & $1(25,50)$ & $1(25,50)$ \\
\hline $\operatorname{PE}(7 / 12 ; 36,8 \%)$ & $7(100,0)$ & $3(42,9)$ & $5(71,4)$ & $5(71,4)$ & $4(57,1)$ & $4(57,1)$ \\
\hline $\mathrm{PI}(3 / 16 ; 18,7 \%)$ & $3(100,0)$ & $1(33,3)$ & $2(66,7)$ & $2(66,7)$ & $2(66,7)$ & $1(33,3)$ \\
\hline $\mathrm{RN}(2 / 11 ; 18,28)$ & $2(100,0)$ & $2(100,0)$ & $2(100,0)$ & $2(100,0)$ & - & $1(50,0)$ \\
\hline $\operatorname{SE}(2 / 9 ; 22,2 \%)$ & $2(100,0)$ & $1(50,0)$ & $1(50,0)$ & $1(50,0)$ & $1(50,0)$ & $1(50,0)$ \\
\hline prabo & & 0,325 & 0,809 & 0,754 & 0,390 & 0,761 \\
\hline
\end{tabular}

\section{Caracterização das instituições nas quais enfermeiras obstetras egressas dos CEEO atuavam}

0 objetivo dos CEEO, com o aprimoramento cultural dos enfermeiros obstétricos, se consubstanciava na premiação da Instituição que implantasse novos modelos assistenciais. Por esse motivo, buscou-se, na segunda fase do estudo, identificar junto aos egressos a participação da instituição em que trabalhavam nos processos de premiação. No Gráfico 1, observa-se que dos 127 egressos, $43(33,9 \%)$ responderam afirmativamente, $77(60,6 \%)$ negaram e $7(5,5 \%)$ não responderam. Dentre os prêmios referidos pelos 43 egressos, aos quais suas instituições de trabalho concorreram ou receberam, foram citados: Hospital Amigo da Criança (20;46,5\%), Implantação do Parto Humanizado e Doulas (4; 9,3\%), Prêmio Galbade Araújo (3; 6,9\%), Mãe Canguru (3; 6,9\%), Prêmios Galba e Hospital Amigo da Criança (2; 4,7\%), Banco de Leite Humano (2; 4,7\%), Prêmio Bibi Vogel (2;4,7\%), Projeto Nascer (1;2,3\%) e Hospital Amigo da Criança e Projeto $\operatorname{Nascer}(1 ; 2,3 \%)$.Este resultado deve ser considerado positivo, uma vez que $33,9 \%$ dos egressos conheciam os prêmios ligados a sua área de atuação, alguns já haviam tido oportunidade de implantar este modelo e mesmo de participar e receber prêmios, além de, consequentemente, saberem quais condutas deveriam ser implantadas no serviço e como deveria ser sua atuação para alcançar esses objetivos. Os 77 egressos que referiram trabalhar em instituição não participante do processo de premiação do Ministério da Saúde forneceram justificativas bem distintas para o fato. Quinze (19,4\%) alegaram não saber informar, $10(13,0 \%)$ não haviam implantado qualquer modelo e $5(6,5 \%)$ informaram haver falta de interesse dos gestores.

Estas respostas indicaram que a qualificação não pareceu ser suficiente para que a atuação do enfermeiro obstetra possibilitasse influenciar comportamentos ou exercer pressão, de forma a fazer a diferença em uma instituição de saúde. Constatou-se que, dentre as instituições participantes ou contempladas com premiações por terem implantado novos modelos assistenciais, 39,8\% tinham egressos atuando na área da saúde da mulher, contrapondo-se a 6,3\% que não dispunham desse corpo funcional, diferença que se mostrou estatisticamente significante. A significância da diferença permite aventar a hipótese de que os egressos fizeram a diferença nos serviços de saúde quando atuaram na sua área de especialização, promovendo modificações no processo assistencial, muito mais que na estrutura, o que resultou nas premiações.

0 Ministério da Saúde avalia as instituições de saúde e indica aquelas que podem ser premiadas, por modificarem o processo de assistência para contemplar a humanização 
do atendimento à gestante e ao recém-nascido, e estimularem o parto normal com alojamento conjunto e o aleitamento materno. Determina então as que efetivamente recebem a premiação. Dessa forma, a associação entre ter egressas do CEEO e ter indicação à premiação ou ser a instituição premiada indica que os CEEO contribuíram na melhoria da assistência em decorrência da atuação dessas egressas.

Figura 1- Distribuição de instituições segundo premiação por implantação de novos modelos assistenciais e presença de egressas do CEEO - Região Nordeste, Brasil, 2006-2007.

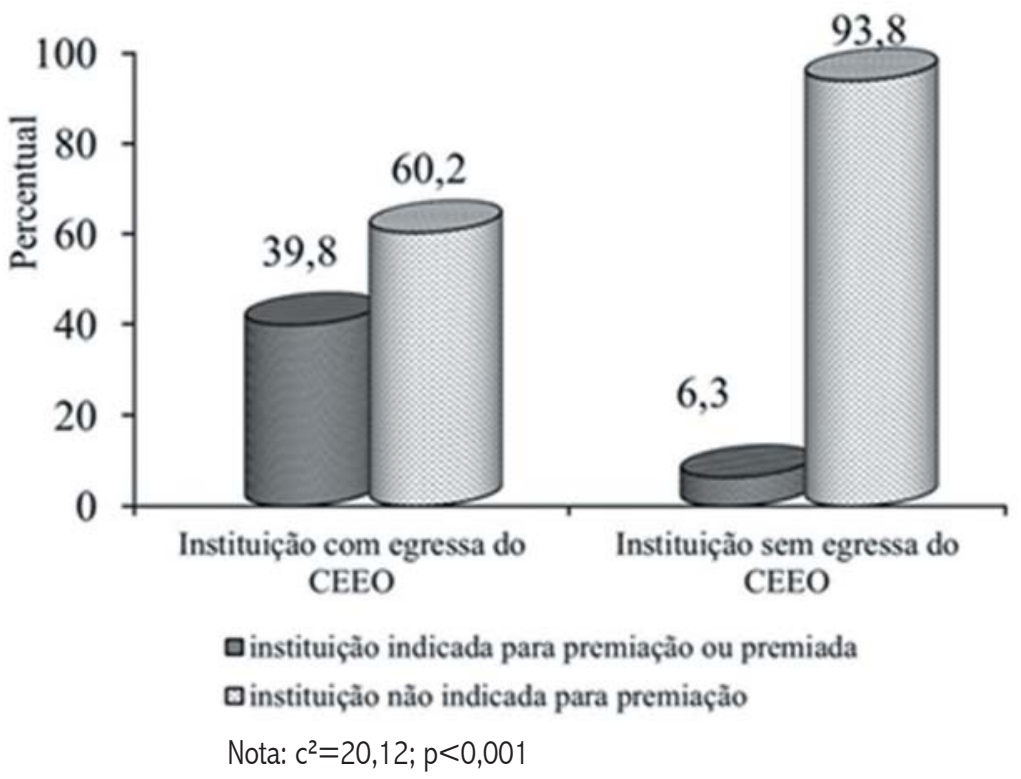

\section{CONCLUSÃO}

Ainda que se considere a limitação metodológica de ter sido empregada amostra de conveniência composta por voluntários, portanto não probabilística, esta pesquisa permitiu identificar que o CEEO pode ter contribuído para a melhoria da assistência obstétrica, já que a maioria dos egressos manteve-se na atividade profissional assistencial, bem como pode ter contribuído indiretamente para a formação de novos profissionais obedecendo à proposta do PHPN, visto que parte dos egressos atuava nos cursos de graduação em enfermagem, ministrando conteúdos relativos à área da mulher em geral. Adicionalmente, essa formação possibilitou a implantação de novos modelos assistenciais, que se consubstanciaram na participação do processo de premiação ou na premiação das instituições nas quais trabalhavam (Figura 1)

\section{REFERÊNCIAS}

1. Mac Donald M, Starrs A. La atención calificada durante el parto: un cuaderno informativo para salvar la vida de las mujeres y mejorar la salud de los recién nascidos. New York: Family Care International; 2003.

2. World Health Organization- WHO. Appropriate technology for birth. Lancet 1985 Aug; 8452: 436-37.
3. Diniz CSG. Humanization of childbirth care in Brazil: the numerous meanings of a movement. Cienc Saude. 2005 jul/set; 10(3): 627-37.

4. Ministério da Saúde (BR). Secretaria de Políticas de Saúde. Área Técnica de Saúde da Mulher. Comitê de Especialistas em Enfermagem Obstétrica: diretrizes para elaboração de projetos de cursos de especialização em enfermagem obstétrica. Brasília (DF);2004.

5. Ministério da Saúde (BR). Portaria n. 570,A de $1^{\circ}$ de junho de 2000. Institui o Componente I do PHPN - Incentivo à assistência pré-natal. Diário Oficial da República Federativa do Brasil, Brasília (DF), 8 de junho de 2000, seção 1:8.

6. Ministério da Saúde (BR). Portaria $n^{0}$ 571, de $1^{\circ}$ de junho de 2000. Institui o Componente II do PHPN - Organização, Regulação e Investimentos na Assistência Obstétrica e Neonatal. Diário Oficial da República Federativa do Brasil, Brasília (DF), 8 de junho de 2000, seção 1:8.

7. Ministério da Saúde (BR). Portaria $n^{0} 572$ de $1^{\circ}$ de junho de 2000. Institui o Componente III do PHPN - Nova Sistemática de Pagamento à Assistência ao Parto. Diário Oficial da República Federativa do Brasil, Brasilia (DF), 8 de jun 2000; Seção 1:9.

8. Ministério da Saúde (BR). Secretaria de Políticas de Saúde. Área Técnica de Saúde da Mulher. Relatório da reunião de avaliação dos cursos de especialização em enfermagem obstétrica. Brasília(DF); 2002.

9. Ministério da Saúde (BR). Secretaria de Atenção a Saúde. Departamento de Ações Programáticas Estratégicas. Área Técnica de Saúde da Mulher. Relatório de Gestão 2003-2006. Brasília(DF); 2007. 
10. Instituto Brasileiro de Geografia e Estatística- IBGE. Estatísticas da Saúde: assistência médico-hospitalar. Brasília(DF);2005. 300p.

11. Schirmer J. Formação da enfermeira obstetra: contribuição para o resgate da atuação na assistência ao parto e nascimento. [tese de doutorado] Rio de Janeiro: Escola de Enfermagem/USP; 2001.

12. Riesco MLG. Perspectivas para formação e qualificação profissional para assistência ao nascimento e parto no nível de graduação. $2^{\circ}$ Seminário Estadual sobre o Ensino de Enfermagem para Assistência ao Parto e Nascimento; 1999; Sorocaba : ABENFO-SP/PUC-SP; 1999. p. 59-75.

13 Ministério da Saúde (BR). Instituto Nacional de Estudos e Pesquisas Educacionais Anísio Teixeira-INEP. In: Dilvo Ristoff, organizador. A mulher na educação brasileira: 1991-2005. Brasília (DF); 2007.

14. Merighi MAB. Enfermeiras obstétricas egressas da Escola de Enfermagem da Universidade de São Paulo: caracterização e trajetória profissional. [tese de doutorado]. São Paulo: Escola de Enfermagem/ USP; 2005.

15. Clapis MJ. Competências de enfermeiras obstétricas na atenção qualificada ao parto: contribuições do Curso de Especialização em Enfermagem Obstétrica e Neonatal - modalidade residência. [tese de doutorado]. São Paulo: Escola de Enfermagem de Ribeirão Preto/ USP; 2005.

16. Riesco MLG, Tsunechiro MA. Formação profissional de obstetrizes e enfermeiras obstétricas: velhos problemas ou novas possibilidades? Est Fem. 2002 jul/dez; 2: 449-59.

17. Andrade V, Padilha KG, Kimura M. Segmento dos enfermeiros egressos dos cursos de especialização em enfermagem em cuidados intensivos. Rev Latino-Am Enfermagem. 1998 jul; 6(3): 23-31.

18. Rocha TA, Bonilha ALL. Formação de enfermeiras para a parturição: implantação de um hospital universitário na década de 80. Esc Anna Nery. 2008 dez;12(4): 651-57. 